\title{
A Plasma Protein Biomarker Strategy for Detection of Small Intestinal Neuroendocrine Tumors
}

\author{
Magnus Kjellman ${ }^{a}$ Ulrich Knigge ${ }^{b}$ Staffan Welin ${ }^{c}$ Espen Thiis-Evensen ${ }^{d}$ \\ Henning Gronbaek ${ }^{e}$ Camilla Schalin-Jäntti ${ }^{f}$ Halfdan Sorbye $^{g}$ \\ Maiken Thyregod Joergensen ${ }^{\mathrm{h}}$ Viktor Johanson $^{\mathrm{i}}$ Saara Metso $^{\mathrm{j}}$ Helge Waldum $^{\mathrm{k}}$ \\ Jon Arne Søreide ${ }^{l}$ Tapani Ebeling $^{\mathrm{m}} \quad$ Fredrik Lindberg $^{\mathrm{n}} \quad$ Kalle Landerholm $^{\circ}$ \\ Goran Wallin $^{p}$ Farhad Salem $^{q}$ Maria del Pilar Schneider ${ }^{r}$ Roger Belusa ${ }^{s}$ \\ ${ }^{a}$ Endocrine Surgery Unit, Karolinska Hospital, Stockholm, Sweden; ${ }^{b}$ Department of Endocrinology and Gastrointestinal \\ Surgery, ENETS Neuroendocrine Tumor Centre of Excellence, Rigshospitalet, University of Copenhagen, Copenhagen, \\ Denmark; ' Department of Endocrine Oncology, ENETS Neuroendocrine Tumor Centre of Excellence, Uppsala

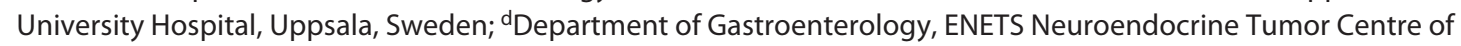

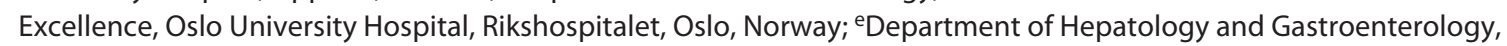 \\ ENETS Neuroendocrine Tumor Centre of Excellence, Aarhus University Hospital, Aarhus, Denmark; fDepartment \\ of Endocrinology, Abdominal Center, University of Helsinki and Helsinki University Hospital, Helsinki, Finland; \\ 9Department of Oncology and Department of Clinical Science, Haukeland University Hospital, Bergen, Norway; \\ hDepartment of Medical Gastroenterology and Hepatology, Odense University Hospital, Odense, Denmark; \\ iDepartment of Surgery, Institute of Clinical Sciences at the Sahlgrenska Academy, University of Gothenburg, \\ Gothenburg, Sweden; Unit of Endocrinology, Department of Internal Medicine, Tampere University Hospital, \\ Teiskontie Tampere, Tampere, Finland; kSt. Olavs Hospital, Trondheim, Norway; 'Department of Gastrointestinal \\ Surgery, Stavanger University Hospital, Stavanger, Norway; mFaculty of Medicine, University of Oulu, Finland and \\ Division of Endocrinology, Oulu University Hospital, Oulu, Finland; n'Department of Surgery, Norrland University \\ Hospital, Umeå, Sweden; ${ }^{\circ}$ Department of Clinical and Experimental Medicine, Linköping University and Department

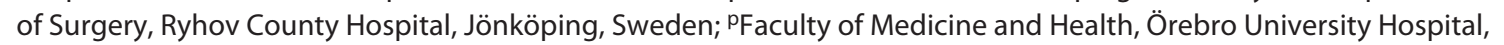 \\ Örebro, Sweden; 'Tkånes University Hospital, Unit for Endocrine and Sarcoma Surgery, Lund, Sweden; \\ 'IPSEN Innovation SAS, Les Ulis, France; SIPSEN, Kista Science Tower, Kista, Sweden
}

\section{Keywords}

Neuroendocrine tumor - Biomarker - Diagnosis · Machine learning

\section{Abstract \\ Background: Small intestinal neuroendocrine tumors (SI- NETs) are difficult to diagnose in the early stage of disease. Current blood biomarkers such as chromogranin A ( $\mathrm{cgA})$}

karger@karger.com www.karger.com/nen Karger" BOPEN ACCESS
(C) 2020 The Author(s)

Published by S. Karger AG, Basel

This is an Open Access article licensed under the Creative Commons Attribution-NonCommercial-4.0 International License (CC BY-NC) (http://www.karger.com/Services/OpenAccessLicense), applicable to the online version of the article only. Usage and distribution for commercial purposes requires written permission. and 5-hydroxyindolacetic acid have low sensitivity (SEN) and specificity (SPE). This is a first preplanned interim analysis (Nordic non-interventional, prospective, exploratory, EXPLAIN study [NCT02630654]). Its objective is to investigate if a plasma protein multi-biomarker strategy can improve diagnostic accuracy (ACC) in SI-NETs. Methods: At the time of diagnosis, before any disease-specific treatment was initiated, blood was collected from patients with advanced SINETs and 92 putative cancer-related plasma proteins from 
135 patients were analyzed and compared with the results of age- and sex-matched controls $(n=143)$, using multiplex proximity extension assay and machine learning techniques. Results: Using a random forest model including 12 top ranked plasma proteins in patients with SI-NETs, the multibiomarker strategy showed SEN and SPE of 89 and $91 \%$, respectively, with negative predictive value (NPV) and positive predictive value (PPV) of 90 and $91 \%$, respectively, to identify patients with regional or metastatic disease with an area under the receiver operator characteristic curve (AUROC) of 99\%. In 30 patients with normal CgA concentrations, the model provided a diagnostic SPE of $98 \%$, SEN of $56 \%$, and NPV $90 \%$, PPV of $90 \%$, and AUROC $97 \%$, regardless of proton pump inhibitor intake. Conclusion: This interim analysis demonstrates that a multi-biomarker/machine learning strategy improves diagnostic ACC of patients with SI-NET at the time of diagnosis, especially in patients with normal CgA levels. The results indicate that this multi-biomarker strategy can be useful for early detection of SI-NETs at presentation and conceivably detect recurrence after radical primary resection.

(c) 2020 The Author(s).

Published by S. Karger AG, Basel

\section{Introduction}

Small intestinal neuroendocrine tumors (SI-NETs) are rare neoplasms with a low but increasing incidence in the range from 0.5 to 1.5 per 100,000 inhabitants per year [1-5]. SI-NETs may be incidentally found during work up of other conditions, or patients may present with carcinoid syndrome (diarrhea and flushing) and with intermittent abdominal pain or acute bowel obstruction. Some of these symptoms are non-specific and often seen in irritable bowel syndrome. Therefore, correct SI-NET diagnosis is often delayed by several years [6]. At the time of diagnosis, many patients present with disseminated disease, making curative surgery impossible [7].

Circulating plasma chromogranin A ( $\mathrm{CgA})$, a glycoprotein released by neuroendocrine cells, and 5-hydroxyindolacetic acid (5-HIAA), a serotonin breakdown product that can be measured in blood and urine, have for decades been used as biomarkers in the diagnosis, treatment response, and follow-up of SI-NETs [8-11]. Neuron-specific enolase, another neuroendocrine related protein, has only shown low to moderate levels of sensitivity (SEN) and specificity (SPE) for GEP-NEN [12].

Most conclude that these biomarkers have relatively low SEN and SPE $[13,14]$. Thus, there is a need for new biomarkers that would accurately detect SI-NETs at an early curable stage, both regarding primary diagnosis as well as for detection of recurrent and progressive disease [13, 15-17].

Newly discovered biomarkers such as miRNA have shown potential to improve the diagnostic SEN and SPE [18-20]. Several other novel biomarkers such as immunemolecules and circulating tumor cells are under investigation [20].

Previous studies in patients with NETs using RNAbased multi-biomarker approaches demonstrated improved tumor classification, metastatic profiling, as well as diagnostic accuracy (ACC) [21-23]. The proximity extension assay (PEA) technology has been used in several cancer studies to identify new biomarker candidates in patients with multiple myeloma [24], colorectal cancer [25], and ovarian cancer [26]. Edfeldt et al. [27] discovered new proteins that correlated with survival in patients with SINETs, using blood samples from 23 patients with SI-NETs that were analyzed with proximity ligation assay and PEA.

Machine learning is a scientific field with origins in computer science, artificial intelligence, and statistics. These techniques were introduced in cancer research $>15$ years ago [28]. The idea is to produce computer algorithms that learn how to correctly perform specific assignments in order to identify certain patterns present in a data set. Random forest (RF) is a well-established statistically supervised learning model and has been used to improve cancer diagnosis in previous studies $[29,30]$. Adam et al. [31] demonstrated that multi-plasma protein analysis in combination with statistical machine learning techniques improved diagnostic ACC of prostate cancer by discriminating it from benign hyperplasia.

The aim of the first interim analysis from the EXPLAIN study was to investigate, in a real-life clinical setting, whether a plasma protein multi-biomarker strategy, measuring the relative concentrations of 92 putative cancer-related human proteins, in combination with machine learning, can provide new biomarkers to improve diagnostic ACC in SI-NETs.

\section{Materials and Methods}

This is an interim analysis from the Nordic EXPLAIN study (exploratory, non-interventional study for evaluating the diagnostic, prognostic, and response-predictive value of a multi-biomarker approach in metastatic GEP NETs [ClinicalTrials.gov: NCT02630654]).

The study was approved by the regional Ethics Committee in each participating country (Denmark, Finland, Norway, and Sweden), and the study complied with the Declaration of Helsinki and 
Good Clinical Practice guidelines. All patients and the control group provided written informed consent after full explanation of the purpose and nature of all procedures used.

\section{Patients}

A total of 175 patients were initially recruited from 17 hospitals and screened to enable 150 patients with SI-NETs to enter the study. Thirty-five patients who were included had surgery prior to inclusion. Inclusion criteria were as follows: provision of written informed consent, suspected metastatic SI-NET (World Health Organization [WHO] grade 1 or 2 , up to $10 \% \mathrm{Ki}-67$ ), and aged 18 years or older. SI-NET diagnosis was confirmed in all patients according to current clinical practice at each hospital. Exclusion criteria were as follows: NET not confirmed, previously treated for NET disease with anti-proliferative treatments, peptide receptor radionuclide therapy or other radiation therapy, other malignant diseases, chronic inflammatory diseases, and severe renal and/or liver disease. Patients who had surgery of the primary tumor but with residual metastatic disease could be included.

Clinical data were collected at each visit and as part of regular patient follow-up and entered into an electronic case report form (Viedoc; Pharma Consulting Group, Uppsala, Sweden). The use of proton pump inhibitors (PPIs) was registered. Patients using PPIs were not excluded, nor was the PPI treatment stopped before blood sample collection. Radiologic evaluation of metastatic disease at the different centers included contrast enhanced computed tomography, MRI, and somatostatin receptor positron emission tomography/computed tomography imaging.

A control group of 155 age- ( \pm 5 years) and sex-matched individuals was included for biomarker blood sampling. Exclusion criteria were as follows: malignant disease, chronic inflammatory disease, and renal or hepatic failure. The individuals were selected from the Karolinska University Hospital Clinical Pharmacology Trial Unit. Three control individuals were excluded due to the presence of chronic inflammatory disease.

\section{Sample Collection and Analysis}

A study blood sample $(4 \mathrm{~mL})$ was collected starting at the first visit and before any NET-specific treatment was initiated. Thereafter, blood samples were collected at visits that were part of the patients' normal follow-up. Samples were prepared by collecting blood in tubes containing ethylenediaminetetraacetic acid and placed on ice immediately after sampling. The samples were centrifuged at 2,500 g for $10 \mathrm{~min}$ at $4^{\circ} \mathrm{C}$. After centrifugation, plasma was aspirated, aliquoted to new tubes $(4 \times 0.5 \mathrm{~mL})$, and immediately hereafter stored at $-80^{\circ} \mathrm{C}$. Blood samples for the exploratory plasma protein biomarkers were transported on dry ice to SciLifeLab, Uppsala, Sweden, and analyzed using multiplex PEA by realtime PCR using the Fluidigm BioMark HD real-time PCR platform with the Olink Proseek Oncology II panel (Olink Proteomics, Uppsala, Sweden, http://www.olink.com/) as previously described $[32,33]$. The multiplex PEA was developed from the proximity ligation assay technique by [32]. One pair of antibodies was used to target each specific protein. The antibodies were coupled to complementary oligonucleotides enabling DNA polymerase to amplify the double-stranded DNA, generating a PCR-reporter sequence by the proximity-dependent DNA polymerization event. The panel of proteins is selected by experts at Olink Proteomics after fulfilling several technical as well as biological criteria. The panel was selected on the basis that these proteins previously have
Table 1. Clinical characteristics for all patients with SI-NETs and for patients with SI-NETs and CgA $\leq \mathrm{ULN}(\leq 5.3 \mathrm{nmol} / \mathrm{L})$ at the time of diagnosis

\begin{tabular}{|c|c|c|}
\hline & $\begin{array}{l}\text { All patients } \\
\text { with SI-NETs } \\
(n=135)\end{array}$ & $\begin{array}{l}\text { Patients with } \\
\text { SI-NETs and } \mathrm{CgA} \leq \\
\mathrm{ULN}(\leq 5.3 \mathrm{nmol} / \mathrm{L}) \\
(n=30)\end{array}$ \\
\hline \multicolumn{3}{|c|}{ Primary tumor location, $n(\%)$} \\
\hline Small intestine & $122(90.4)$ & $27(90.0)$ \\
\hline Unknown & $13(9.6)$ & $3(10.0)$ \\
\hline \multicolumn{3}{|c|}{ Regional lymph nodes, $n(\%)$} \\
\hline $\mathrm{Nx}$ & $10(7.4)$ & $0(0)$ \\
\hline No & $5(3.7)$ & $7(23.3)$ \\
\hline N1 & $120(88.9)$ & $23(76.7)$ \\
\hline \multicolumn{3}{|c|}{ Distant metastases, $n(\%)$} \\
\hline $\mathrm{Mx}$ & $13(9.6)$ & $1(3.3)$ \\
\hline M0 & $31(23.0)$ & $20(66.6)$ \\
\hline M1 & $91(67.4)$ & $9(30.0)$ \\
\hline \multicolumn{3}{|l|}{ NET grade, $n(\%)$} \\
\hline Missing & $5(3.7)$ & $3(10.0)$ \\
\hline G1 & $61(45.2)$ & $17(56.7)$ \\
\hline $\mathrm{G} 2$ & $69(51.1)$ & $10(33.3)$ \\
\hline \multicolumn{3}{|l|}{ No. of metastases } \\
\hline Mean (SD) & $9.4(8.6)$ & $4.2(4.2)$ \\
\hline Median (range) & $7(1-36)$ & $2(1-13)$ \\
\hline \multicolumn{3}{|l|}{$\mathrm{Ki}-67 \%$} \\
\hline Mean (SD) & $4.8(4.1)^{\mathrm{a}}$ & $1.8(2.7)^{\mathrm{C}}$ \\
\hline Median (range) & $4(1-17)^{\mathrm{a}}$ & $1.5(0.3-10)^{\mathrm{c}}$ \\
\hline \multicolumn{3}{|l|}{ Plasma CgA, nmol/L } \\
\hline Mean (SD) & $46.8(85.7)$ & $3.7(1.1)$ \\
\hline Median (range) & $48(2-620)$ & $3.6(2.0-5.3)$ \\
\hline \multicolumn{3}{|c|}{ Urine/serum 5-HIAA (\%ULN) } \\
\hline Median (range) & $168(16-5,953)^{b}$ & $73(16-148)^{\mathrm{d}}$ \\
\hline
\end{tabular}

CgA, chromogranin A; ULN, upper limit of normal; SI-NET, small intestinal neuroendocrine tumor. ${ }^{\mathrm{a}} n=126 .{ }^{\mathrm{b}} n=83{ }^{\mathrm{c}} n=$ 26. ${ }^{\mathrm{d}} n=21$.

been shown to be associated with mechanisms in neoplastic disease and classified according to UniProt, Human Protein Atlas, Gene Ontology, and DisGeNET [24, 25].

Chromogranin A was analyzed centrally at Akademiska Laboratoriet, Uppsala, Sweden, with the NEOLISA ${ }^{\mathrm{TM}}$ Chromogranin A assay (Euro Diagnostica). Urine/serum 5-HIAA was analyzed at each individual hospital using methods according to clinical routine and presented as percent of upper limit of normal (\%ULN). Serum 5-HIAA was measured at 3 clinics in Finland, morning urine 5-HIAA was used in 1 clinic in Norway, and 13 clinics used 24-h urine samples. A total of 135 patients with SI-NETs and 143 age- and sex-matched controls with complete biomarker information were retained for the data analysis.

\section{Statistical Analysis}

Levels of biomarkers were compared between patients with SINETs and the control group. Folded $F$ test was used to test for evidence of equal variance between the 2 populations. If the results 
Table 2. Performance metrics generated with random forest for model 1 and model 2

\begin{tabular}{|c|c|c|c|c|c|c|}
\hline & \multicolumn{4}{|l|}{ Model 1} & \multirow{2}{*}{\multicolumn{2}{|c|}{$\begin{array}{l}\text { Model } 2 \\
\text { including all } 92 \text { biomarkers }\end{array}$}} \\
\hline & \multicolumn{2}{|c|}{ including all 92 biomarkers and CgA } & \multicolumn{2}{|c|}{ including 12 top biomarkers } & & \\
\hline & mean (SD), \% & $\min -\max , \%$ & mean (SD), \% & $\min -\max , \%$ & mean (SD), \% & $\min -\max , \%$ \\
\hline PPV & $90(4.4)$ & $85-96$ & $91(6.6)$ & $82-100$ & $90(13.7)$ & $75-100$ \\
\hline NPV & $88(7.1)$ & $83-100$ & $90(5.9)$ & $86-100$ & $90(8.6)$ & $79-100$ \\
\hline SEN & $87(8.1)$ & $81-100$ & $89(6.4)$ & $85-100$ & $56(32.2)$ & $20-100$ \\
\hline SPE & $91(4.7)$ & $86-97$ & $91(6.3)$ & $83-100$ & $98(2.2)$ & $96-100$ \\
\hline ACC & 89 (3.6) & $84-93$ & $90(4.9)$ & $84-96$ & $89(8.1)$ & $78-100$ \\
\hline AUC & $99(0.5)$ & $98-100$ & $99(0.4)$ & $99-100$ & $97(3.0)$ & $90-100$ \\
\hline MR & $11(3.6)$ & $7-16$ & $10(4.9)$ & $4-16$ & $11(8.1)$ & $0-22$ \\
\hline
\end{tabular}

CgA, chromogranin A; PPV, positive predictive value; NPV, negative predictive value; SEN, sensitivity; SPE, specificity; ACC, accuracy; AUC, area under the curve; MR, misclassification rate; model 1, all patients with SINETs $(n=135)$; model 2 , patients with SI-NETs, and CgA $\leq \mathrm{ULN}(\leq 5.3 \mathrm{nmol} / \mathrm{L})(n=30)$.

of folded $F$ indicated the presence of unequal variance, the WelchSatterthwaite $t$ test was applied. Otherwise, the equal variance $t$ test was used.

$\mathrm{RF}$, a commonly used machine learning algorithm to classify data, was the approach retained for the current study. Preliminary machine learning analysis has shown that RF yielded better ACC to classify between the control group and patients with SI-NETs than support vector machine [34].

Two classification analyses were performed. First, model 1 included all the selected participants, that is, 135 patients with SINETs and 143 the control group, the 92 plasma protein biomarkers, and CgA. CgA values were log-transformed to match the already log-transformed biomarkers. The second RF analysis (model 2) included the control group $(n=143)$ and patients with SI-NETs with CgA values $\leq \mathrm{ULN}(n=30)$. CgA-log was excluded from the analysis since the marker has normal values for the 2 groups of patients.

To validate the RF algorithm, a 5-fold cross-validation was performed. Thus, the complete data set was partitioned into equally (or near equally) sized folds or segments. This gave an approximate split between training and validation sets of 80 and $20 \%$, respectively. The 5-fold cross-validation strategy was chosen to avoid having small groups of patients in the validation sets. This cross-validation strategy was applied to all the models. Software JMP ${ }^{\circledR}$ Pro 14.1.0 and the random forest boosted tree method were used to perform the classification analysis. The classifier constructs a predictive model by adding a sequence of decision trees where each of the trees is fit on the residuals of the previous tree $\left(\mathrm{JMP}^{\circledR} 14\right.$ Predictive and Specialized Modeling 2018, SAS Institute Inc., Cary, NC, USA). A maximum of 50 layers and 3 splits per tree were allowed. Performance of the different models was evaluated by comparing the following metrics: negative predictive value (NPV), positive predictive value (PPV), SEN, SPE, and ACC. These metrics were calculated from the confusion matrix at each fold of the 5-fold cross-validation. Descriptive statistics, that is, mean, standard deviation, and minimum and maximum values, were calculated for each of the performance metrics. Misclassification rate, the rate for which the response with the highest fitted probability is not the observed category, is also provided. Receiver operating characteristic curves and area under the curve were calculated with the software
Python 3.7.0 (package: sklearn). The top proteins were selected after identifying most frequent biomarkers, in 2 or more folds, in the 5 -fold cross-validation in the first random forest analysis.

\section{Results}

\section{Baseline Characteristics}

Of the 175 screened patients, the final SI-NET cohort that fulfilled the inclusion and exclusion criteria had complete biomarker data and was included in the data analyses that consisted of 135 patients. Clinical characteristics of these 135 patients with SI-NETs are given in Table 1.

Median age of all patients was 66 years (range 38-89) and 79 (59\%) were males. Median age of the control group was 67 years (range 36-84) and $83(58 \%)$ were males. In patients $(n=30)$ with CgA concentrations equal or $\leq \mathrm{ULN}$, median age was 66 years (range 44-84) and 20 (66\%) were males.

NET-related symptoms registered at diagnosis in patients with SI-NETs were as follows: 20 (15\%) had >3 daily bowel movements, 10 (7\%) had $>3$ daily flushes, 13 (10\%) had $>3$ daily episodes of abdominal pain, and 35 (26\%) had at least 1 of the 3 symptoms described above. In comparison, in the patient population with plasma CgA $\leq$ ULN ( $n=30)$, symptoms were as follows: $3(10 \%)$ had $>3$ daily bowel movements, 1 (3\%) had $>3$ daily flushes, 2 (7\%) had $>3$ daily episodes of abdominal pain, and 6 (20\%) had at least 1 of the 3 symptoms described above.

\section{Plasma Chromogranin A and 5-HIAA}

Median circulating plasma CgA concentration in the control group was $3.3 \mathrm{nmol} / \mathrm{L}$ (range $2-43$ ). The control group provided a 97 th percentile value at $5.3 \mathrm{nmol} / \mathrm{L}$. A 
Fig. 1. a-c Box plots of the random forest performances (PPV, NPV, SEN, SPE, and ACC) in a 5-fold cross-validation including all patients, 92 plasma protein biomarkers, and CgA-log (model 1, [a]), including all patients and the 12 most frequently identified biomarkers (b) and including the control group and patients with SI-NETs and low CgA ( $\leq$ ULN), 92 biomarkers, and excluding the CgA-log (model 2, [c]). PPV, positive predictive value; NPV, negative predictive value; SEN, sensitivity; SPE, specificity; ACC, accuracy; CgA, chromogranin A; SI-NET, small intestinal neuroendocrine tumor.
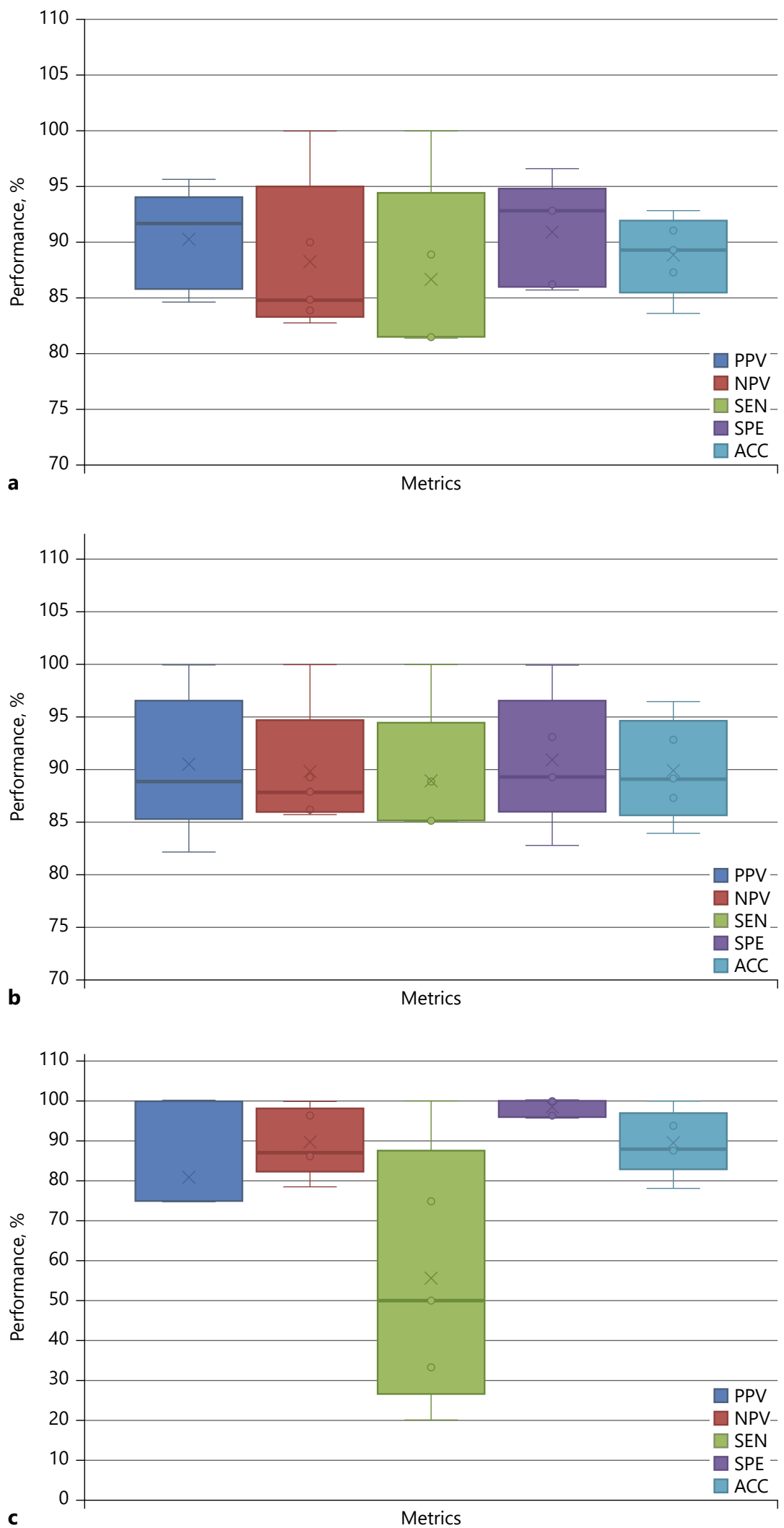

C

Metrics 
cutoff value of $5.3 \mathrm{nmol} / \mathrm{L}$ results in an SEN and SPE for CgA alone similar to what has been shown in other studies (see below).

Using the 97th percentile (i.e., upper limit of normal, $\leq \mathrm{ULN}$ ) as the limit for normal CgA, $22 \%$ of patients ( $n=$ 30) had CgA concentrations below or equal to this value at the time of diagnosis. Patients with $\mathrm{CgA} \leq \mathrm{ULN}$ also showed a lower number of metastasis (median 2, range 1-13) compared with the whole SI-NET patient population (median 7, range 1-36). Of the patients with $\mathrm{CgA} \leq$ ULN, 9 had 1 metastasis (1 patient had 1 liver metastasis and 8 patients had 1 lymph node metastasis only). Plasma CgA concentrations in all patients with SI-NETs and those with $\mathrm{CgA} \leq \mathrm{ULN}$ are presented in Table 1 .

In this study, the mean (SD) diagnostic SEN was 79\% $( \pm 7)$, SPE was $87 \%( \pm 5)$, PPV was $85 \%( \pm 5)$, and NPV was $81 \%( \pm 5)$ for CgA alone. At the time of diagnosis, $12(8 \%)$ were in the control group, $21(16 \%)$ of the patients with SI-NETs, and $3(10 \%)$ of the patients with CgA $\leq$ ULN were treated with PPIs. Urine/serum 5-HIAA levels were increased (>100\% ULN) in $59(71 \%)$ of the 83 patients providing samples within 3 months after the first visit.

\section{Machine Learning Analysis}

RF performances (model 1) evaluated as NPV, PPV, SEN, and SPE in a 5 -fold cross-validation (mean [SD]) were $88( \pm 7), 90( \pm 4), 87( \pm 8)$, and $91 \%( \pm 5)$, respectively. Table 2 shows all the descriptive statistics for the performance metrics evaluated in model 1 . Figure 1a summarizes the 5-fold cross-validation results for model $1 \mathrm{ex}$ pressed as PPV, NPV, SEN, SEN, and ACC. The graph aims to show the variability of the model's metrics across the different folds. Using all 92 biomarkers and CgA, the model did not discriminate between grade 1 and 2 at the time of diagnosis.

An additional RF analysis was performed including only the biomarkers with the highest contribution to model 1. Thus, a total of 12 biomarkers, that is, most frequently identified in the 5-fold cross-validation (in 2 or more folds) and having the highest contribution to the classification model, were selected (Table 3).

Mean (SD) SEN and NPV improved slightly by including only the 12 most highly ranked plasma protein biomarkers, from $87( \pm 8)$ to $89 \%( \pm 6)$ and from $88( \pm 7)$ to $90 \%( \pm 6)$, respectively. All the metrics performances are presented in Table 2 and Figure 1b. The area under the receiver operator characteristic curve (AUROC) analysis including only 12 biomarkers identified a mean $( \pm \mathrm{SD})$ AUROC of $99 \%$ ( \pm 0.4 ) (Fig. 2). Model 2 included the control group and patients with SI-NETs and CgA $\leq$ ULN
Table 3. Most frequently identified biomarkers in the 5-fold crossvalidation (in 2 or more folds)

\begin{tabular}{lll}
\hline Biomarker & \multicolumn{2}{l}{ Frequency in 5-fold cross-validation } \\
\cline { 2 - 3 } & model 1 & model 2 \\
\hline CgA-log & 5 & NA \\
LYN & 5 & 5 \\
ITGAV & 4 & 4 \\
CDKN1A & 4 & NI \\
CAIX & 4 & NI \\
ABL1 & 3 & 3 \\
5'-NT & 3 & NI \\
EGF & 3 & 3 \\
PVRL4 & 2 & NI \\
TRAIL & 2 & 2 \\
VIM & 2 & 4 \\
MUC-16 & 2 & NI \\
CRNN & NI & 3 \\
VEGFA & NI & 3 \\
CD70 & NI & 3 \\
hK14 & NI & 2 \\
S100A11 & NI & 2 \\
\hline
\end{tabular}

Information on each protein can be found at https://www.uniprot.org. NA, not applicable; NI, not identified; model 1, all patients with SI-NETs $(n=135)$; model 2, patients with SI-NETs, and $\mathrm{CgA} \leq \mathrm{ULN}(\leq 5.3 \mathrm{nmol} / \mathrm{L})(n=30)$.

$(n=30)$. CgA-log was excluded from the data analysis since the marker has normal values within the groups of patients. The 5-fold cross-validation yielded the following performances (mean [SD]): NPV 90\% ( \pm 9 ), PPV 90\% $( \pm 14)$, SEN $56 \%( \pm 32)$, and SPE $98 \%( \pm 2)$. Additional performance metrics are presented in Table 2. Figure 1c summarizes the 5 -fold cross-validation results for model 2 . Table 3 shows the 12 biomarkers most frequently identified in the 5-fold cross-validation with the highest contribution to the classification model between the control group and patients with SI-NETs and CgA $\leq$ ULN.

\section{Discussion}

To our knowledge, this is the first study that analyzes the relative levels of close to 100 putative cancer-related plasma proteins at the time of diagnosis in a large SI-NET patient population in a real-life clinical setting. In this interim analysis, the main finding is that PEA and combined machine learning techniques improve diagnostic PPV and NPV in patients with SI-NETs at the time of diagnosis, including those with $\mathrm{CgA}$ concentrations 


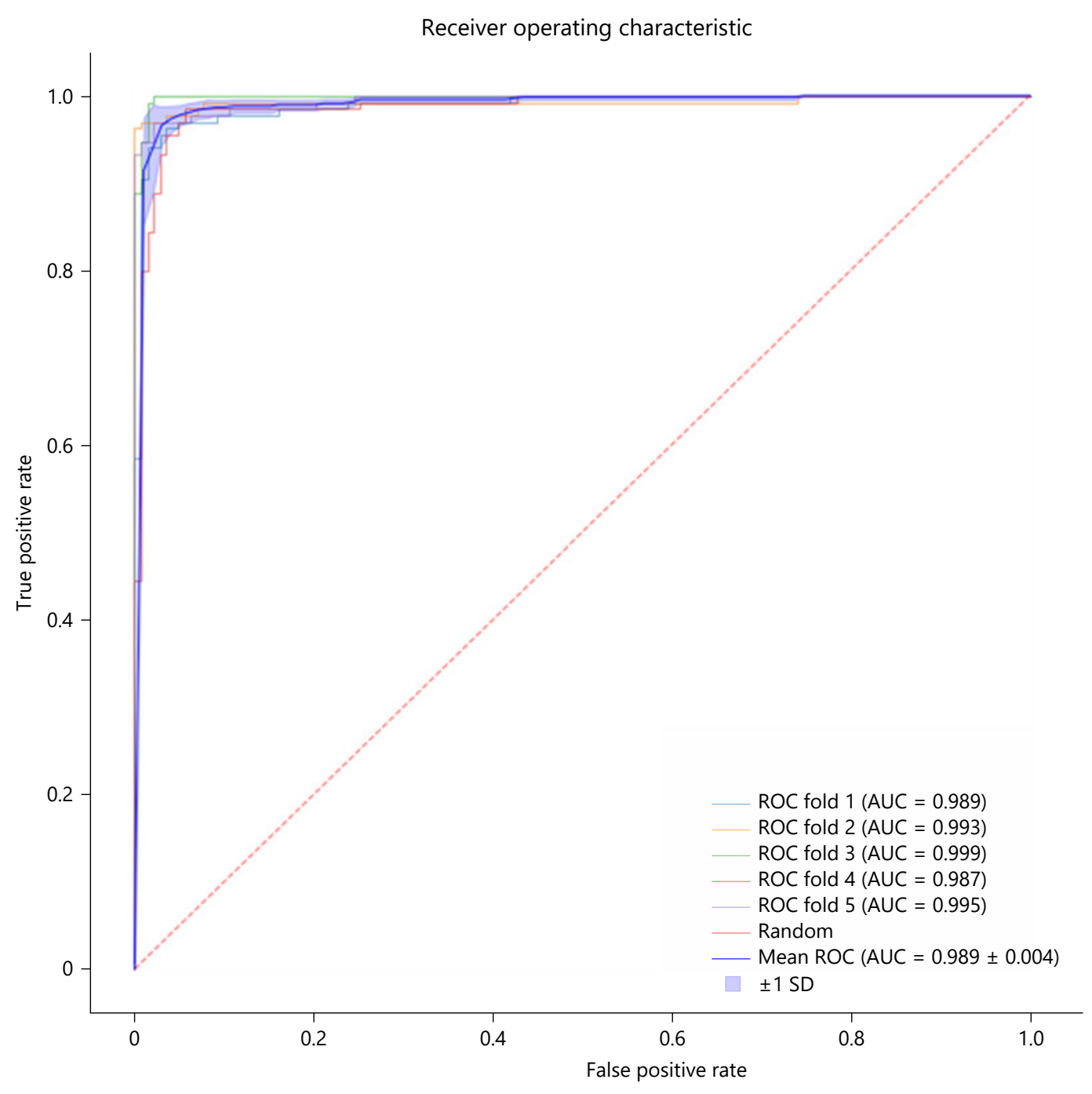

Fig. 2. ROC curves and the corresponding AUC values generated from a random forest model analysis including the control group and patients with SI-NETs and the top 12 ranked identified plasma protein biomarkers. ROC, receiver operating characteristic; AUC, area under the curve.

$\leq \mathrm{ULN}$, providing both a PPV and NPV of $90 \%$. The results imply that this multi-biomarker strategy may be useful for early detection of SI-NETs, both at presentation and to possibly detect recurrence after radical resection of the primary tumor. Methods with better SEN and SPE to detect SI-NETs at an early and curable stage are warranted as blood biomarkers such as CgA, neuron-specific enolase, pancreastatin, and circulating tumor cells so far lack the SEN, SPE, and predictive capacity needed in routine clinical practice [13].
The PEA technology used for measuring plasma proteins in the present study is not yet adapted to and validated for routine clinical practice. However, it is valuable in the discovery phase of research since it generates large amounts of biomarker data. In order to better analyze our data, we used supervised machine learning techniques. This approach clearly out-performs analysis of plasma $\mathrm{CgA}$ alone. Our finding suggests that plasma CgA does not provide sufficient diagnostic ACC as a mono-biomarker in accordance with previous studies $[12,14]$. Of 
note, several factors are known to influence $\mathrm{CgA}$ concentrations, for example, intake of PPIs, renal failure, atrophic gastritis, and hepatic disease [35-37]. Zatelli et al. [38] demonstrated that CgA as a mono-biomarker had an SEN around 0.8 at its best, similar to the findings of the present study.

Importantly, a significant finding of the present study is that our biomarker test improved detection of SI-NETs in patients with regional or metastatic disease who had normal CgA concentrations. This patient subgroup was further characterized by lower concentrations of 5-HIAA, a low Ki-67 index, and low number of metastases compared with patients with verified metastatic disease and elevated CgA concentrations (Table 1). This patient subgroup displays fewer NET-specific symptoms and is the most difficult to diagnose. In our study, mean NPV and PPV for this patient subgroup were as high as $90 \%$, with an AUROC of $97 \%$. Consequently, this method could aid in accurate and timely primary diagnosis. In addition, it could be used to identify early recurrences in the followup of patients after radical surgical resection and eventually also in screening of members in families with increased incidence of SI-NETs [39]. This multi-biomarker strategy could thus decrease the need for expensive and burdensome radiologic examinations.

Our study has several strengths including the "real-life clinical setting" design, the large number of patients with SI-NETs with matched controls, as well as centralized analysis of $\mathrm{CgA}$ and biomarkers. However, this is a first exploratory study and the model has not been tested to discriminate between patients with SI-NETs and non-SINETs with similar symptomatology, such as diarrhea, abdominal pain, and flushing. Future validation studies are needed to clarify these aspects including other primary tumors, for example, pancreatic NET. Furthermore, even if the primary tumor was removed prior to inclusion in 35 patients (as a result of small intestinal surgery), the patients still had advanced disease when they were included. It can, however, be speculated that in some patients this may have impaired the SEN and SPE of the biomarker profile.

A similar approach to that of the present study used an mRNA-based blood multi-biomarker model (multiple transcriptional analyses) and reported data which improved diagnostic ACC, treatment response prediction, as well as prediction of progression [40-42]. The diagnostic ACC (AUROC of 0.95-0.98) of this mRNA-based multi-biomarker model is in the same range as in the present study. A recent Delphi assessment of currently available NET biomarkers concluded that there is an im- minent unmet medical need to develop more informative biomarker tools [13].

Patients who have undergone possible radical resection are 1 group of patients in whom this test could improve diagnostic ACC. The biomarker test could also aid in discriminating those patients that truly can be considered disease-free from those having microscopic remnant disease. This study provides a method for improving early diagnosis in patients with normal plasma CgA, 5-HIAA, and low tumor burden and should be validated in larger SI-NET population in the future. In addition, the SPE of the current biomarker test needs to be investigated in other NET subtypes such as pancreatic and lung NETs.

In conclusion, this is the first pre-planned interim analysis of the Nordic EXPLAIN biomarker study in an SI-NET patient population. The multi-plasma protein analysis strategy improved diagnostic SPE and SEN. Importantly, the model discriminated between healthy individuals and patients with SI-NETs characterized by normal CgA concentrations, moderate disease burden, and mild symptomatology. These biomarkers should be further studied as a possible test for early diagnosis, recurrence, and potentially treatment response in patients with NET.

\section{Acknowledgements}

We would like to thank all the patients who made this study possible through participation in the study. We thank Torbjorn Ström at IPSEN Nordic and Karin Becker (at IPSEN at the time the study was conducted) for study monitoring. We thank Daniel Seisdedos at the Pharma Consulting Group, Uppsala, Sweden, for data management. We thank Dr. Nabil Al-Tawil at the Karolinska University Hospital Clinical Pharmacology Trial Unit, Stockholm, Sweden, for recruiting sex- and age-matched controls. We would, as well, like to thank all additional physicians and nurses at the 17 hospitals that may have contributed to make this study possible and Boris Sanchot from Starclay for providing support to the machine learning data analysis.

\section{Statement of Ethics}

This study was approved by the local Ethics Committees of the Nordic countries, and written informed consent was obtained from all participants.

\section{Conflict of Interest Statement}

M.K.: grants from IPSEN, consulting fees from IPSEN and Novartis Healthcare. U.K.: grants from IPSEN and Novartis Healthcare, consulting fees from IPSEN. E.T.E.: research grants from No- 
vartis, consulting fees from IPSEN, and speaker honoraria from Novartis, Ipsen, and Pfizer. H.G.: research grants from the Novo Nordisk Foundation, Intercept, Abbvie, and consulting fees from IPSEN and Novartis. C.S.J.: grant from IPSEN, consulting fees from IPSEN, Kyowa Kirin, and Novartis. H.S.: received research support from Novartis, Amgen, Ipsen and honoraria from Novartis, Ipsen, Pfizer, Keocyt, AstraZeneca, Roche, Amgen, BMS, Hutchinson, Merck, Shire, and Celgene. H.W.: no conflicts of interest. V.J.: no conflicts of interest. M.T.J.: no conflicts of interest. S.W.: consulting fees from IPSEN and Novartis Healthcare. J.A.S.: consulting fee from Novartis. S.M.: speaker honoraria from Amgen, Ipsen, Novartis, Novo Nordisk, MSD, and Sanofi. T.E.: speaker honoraria from Ipsen, AstraZeneca, and Novo Nordisk Pharma, consulting fee from Amgen, educational grants by Novartis, Ipsen, and MSD Finland to the employer institution for international symposia. F.L.: speaker honorarium from Novartis. K.L.: no conflicts of interest. F.S.: no conflicts of interest. G.W.: no conflicts of interest. M.d.P.S.: IPSEN employee. R.B.: IPSEN employee.

\section{Funding Sources}

This study was funded by IPSEN.

\section{Author Contributions}

Design of the study: M.K., U.K., E.T.E., H.G., C.S.J., H.S., H.W., V.J., M.T.J., S.W., J.A.S., S.M., T.E., F.L., K.L., G.W., F.S., and R.B. Preparing the study protocol: R.B., M.K., U.K., E.T.E., H.G., and C.S.J. Patients inclusion: M.K., U.K., E.T.E., H.G., C.S.J., H.S., H.W., V.J., M.T.J., S.W., J.A.S., S.M., T.E., F.L., K.L., G.W., and F.S. Data management: Pharma Consulting Group, Uppsala, Sweden, and R.B. Statistical analysis: M.d.P.S. Preparation of manuscript: R.B., M.d.P.S., M.K., U.K., E.T.E., H.G., and C.S.J. All authors have reviewed/edited the manuscript and approved the final version for submission.

\section{Data Sharing}

Where patient data can be anonymized, Ipsen will share all individual participant data that underlie the results reported in this article with qualified researchers who provide a valid research question. Study documents, such as the study protocol and clinical study report, are not always available. Proposals should be submitted to DataSharing@Ipsen.com and will be assessed by a scientific review board. Data are available beginning 6 months and ending 5 years after publication; after this time, only raw data may be available.

\section{References}

1 Yao JC, Hassan M, Phan A, Dagohoy C, Leary C, Mares JE, et al. One hundred years after "carcinoid": epidemiology of and prognostic factors for neuroendocrine tumors in 35,825 cases in the United States. J Clin Oncol. 2008 Jun;26(18):3063-72.

2 Lawrence B, Gustafsson BI, Chan A, Svejda B, Kidd M, Modlin IM. The epidemiology of gastroenteropancreatic neuroendocrine tumors. Endocrinol Metab Clin North Am. 2011;40(1):1-18,

3 Fraenkel M, Kim M, Faggiano A, de Herder WW, Valk GD; Knowledge NETwork. Incidence of gastroenteropancreatic neuroendocrine tumours: a systematic review of the literature. Endocr Relat Cancer. 2014;21(3): R153-63.

4 Cetinkaya RB, Aagnes B, Thiis-Evensen E, Tretli S, Bergestuen DS, Hansen S. Trends in incidence of neuroendocrine neoplasms in Norway: a report of 16,075 cases from 1993 through 2010. Neuroendocrinology. 2017;104(1):1-10.

5 Dasari A, Shen C, Halperin D, Zhao B, Zhou $\mathrm{S}, \mathrm{Xu} \mathrm{Y}$, et al. Trends in the incidence, prevalence, and survival outcomes in patients with neuroendocrine tumors in the United States. JAMA Oncol. 2017 Oct;3(10):1335-42.

6 Janson ET, Sorbye H, Welin S, Federspiel B, Grønbæk H, Hellman P, et al. Nordic guidelines 2014 for diagnosis and treatment of gastroenteropancreatic neuroendocrine neoplasms. Acta Oncol. 2014 Oct;53(10):1284-97.

7 Hallet J, Law CH, Cukier M, Saskin R, Liu N, Singh S. Exploring the rising incidence of neuroendocrine tumors: a population-based anal- ysis of epidemiology, metastatic presentation, and outcomes. Cancer. 2015;121(4):589-97.

8 O'Connor DT, Deftos LJ. Secretion of chromogranin A by peptide-producing endocrine neoplasms. N Engl J Med. 1986;314(18): $1145-51$.

9 Deftos LJ. Chromogranin A: its role in endocrine function and as an endocrine and neuroendocrine tumor marker. Endocr Rev. 1991 May;12(2):181-7.

10 Nobels FRE, Kwekkeboom DJ, Coopmans W, Schoenmakers CHH, Lindemans J, De Herder WW, et al. Chromogranin A as serum marker for neuroendocrine neoplasia: comparison with neuron-specific enolase and the a-subunit of glycoprotein hormones. J Clin Endocrinol Metab. 1997;82(8):2622-8.

11 Kanakis G, Kaltsas G. Biochemical markers for gastroenteropancreatic neuroendocrine tumours (GEP-NETs). Best Pract Res Clin Gastroenterol. 2012;26(6):791-802.

12 Oberg K, Modlin IM, De Herder W, Pavel M, Klimstra D, Frilling A, et al. Consensus on biomarkers for neuroendocrine tumour disease. Lancet Oncol. 2015;16(9):e435-46.

13 Oberg K, Krenning E, Sundin A, Bodei L, Kidd M, Tesselaar M, et al. A Delphic consensus assessment: imaging and biomarkers in gastroenteropancreatic neuroendocrine tumor disease management. Endocr Connect. 2016;5(5):174-87.

14 Dam G, Grønbæk H, Sorbye H, Thiis Evensen E, Paulsson B, Sundin A, et al. Prospective study of chromogranin A as a predictor of progression in patients with pancreatic, small-intestinal, and unknown primary neuroendocrine tumors. Neuroendocrinology. 2020;110(3-4):217-24.

15 Janson ET, Holmberg L, Stridsberg M, Eriksson B, Theodorsson E, Wilander E, et al. Carcinoid tumors: analysis of prognostic factors and survival in 301 patients from a referral center. Ann Oncol. 1997;8(7):685-90.

16 Welin S, Stridsberg M, Cunningham J, Granberg D, Skogseid B, Oberg K, et al. Elevated plasma chromogranin $\mathrm{A}$ is the first indication of recurrence in radically operated midgut carcinoid tumors. Neuroendocrinology. 2009;89(3):302-7.

17 Kidd M, Bodei L, Modlin IM. Chromogranin A: any relevance in neuroendocrine tumors? Curr Opin Endocrinol Diabetes Obes. 2016; 23(1):28-37.

18 Heverhagen AE, Legrand N, Wagner V, Fendrich V, Bartsch DK, Slater EP. Overexpression of microRNA miR-7-5p is a potential biomarker in neuroendocrine neoplasms of the small intestine. Neuroendocrinology. 2018;106(4):312-7.

19 Vicentini C, Fassan M, D’Angelo E, Corbo V, Silvestris N, Nuovo GJ, et al. Clinical application of microRNA testing in neuroendocrine tumors of the gastrointestinal tract. Molecules. 2014 Feb;19(2):2458-68.

20 Herrera-Martínez AD, Hofland LJ, Gálvez Moreno MA, Castaño JP, de Herder WW, Feelders RA. Neuroendocrine neoplasms: current and potential diagnostic, predictive and prognostic markers. Endocr Relat Cancer. 2019 Mar;26(3):R157-79. 
21 Li SC, Essaghir A, Martijn C, Lloyd RV, Demoulin JB, Öberg K, et al. Global microRNA profiling of well-differentiated small intestinal neuroendocrine tumors. Mod Pathol. 2013;26(5):685-96.

22 Modlin IM, Kidd M, Bodei L, Drozdov I, Aslanian $\mathrm{H}$. The clinical utility of a novel bloodbased multi-transcriptome assay for the diagnosis of neuroendocrine tumors of the gastrointestinal tract. Am J Gastroenterol. 2015; 110(8):1223-32.

23 Bowden M, Zhou CW, Zhang S, Brais L, Rossi A, Naudin L, et al. Profiling of metastatic small intestine neuroendocrine tumors reveals characteristic miRNAs detectable in plasma. Oncotarget. 2017;8(33):54331.

24 Schneiderova P, Pika T, Gajdos P, Fillerova R, Kromer P, Kudelka M, et al. Serum protein fingerprinting by PEA immunoassay coupled with a pattern-recognition algorithms distinguishes MGUS and multiple myeloma. Oncotarget. 2017;8(41):69408.

25 Mahboob S, Ahn SB, Cheruku HR, Cantor D, Rennel E, Fredriksson S, et al. A novel multiplexed immunoassay identifies CEA, IL-8 and prolactin as prospective markers for Dukes' stages A-D colorectal cancers. Clin Proteomics. 2015;12(1):10.

26 Boylan KLM, Geschwind K, Koopmeiners JS, Geller MA, Starr TK, Skubitz APN. A multiplex platform for the identification of ovarian cancer biomarkers. Clin Proteomics. 2017; 14(1):34.

27 Edfeldt K, Daskalakis K, Bäcklin C, Norlén O, Tiensuu Janson E, Westin G, et al. DcR3, TFF3, and midkine are novel serum biomarkers in small intestinal neuroendocrine tumors. Neuroendocrinology. 2017; 105(2): $170-81$.
28 Furey TS, Cristianini N, Duffy N, Bednarski DW, Schummer M, Haussler D. Support vector machine classification and validation of cancer tissue samples using microarray expression data. Bioinformatics. 2000;16(10): 906-14.

29 Glotsos D, Tohka J, Ravazoula P, Cavouras D, Nikiforidis G. Automated diagnosis of brain tumours astrocytomas using probabilistic neural network clustering and support vector machines. Int J Neural Syst. 2005;15(1-2):111.

30 Statnikov A, Wang L, Aliferis CF. A comprehensive comparison of random forests and support vector machines for microarraybased cancer classification. BMC Bioinformatics. 2008 Jul;9:319.

31 Adam BL, Qu Y, Davis JW, Ward MD, Clements MA, Cazares LH, et al. Serum protein fingerprinting coupled with a pattern-matching algorithm distinguishes prostate cancer from benign prostate hyperplasia and healthy men. Cancer Res. 2002 Jul;62(13):3609-14.

32 Lundberg $M$, Thorsen SB, Assarsson E, Villablanca A, Tran B, Gee N, et al. Multiplexed homogeneous proximity ligation assays for high-throughput protein biomarker research in serological material. Mol Cell Proteomics. 2011;10(4):M110.

33 Assarsson E, Lundberg M, Holmquist G, Björkesten J, Thorsen SB, Ekman D, et al. Homogenous 96-plex PEA immunoassay exhibiting high sensitivity, specificity, and excellent scalability. PLoS One. 2014 Apr;9(4):e95192.

34 Kjellman MM, Welin S, Knigge U, Gronbaek $\mathrm{H}$, Sorbye $\mathrm{H}$, Thiis-Evensen E, et al. 463PPlasma protein fingerprinting and machine learning for the diagnosis of small intestinal neuroendocrine tumors: the nordic NET biomarker group EXPLAIN study. Ann Oncol. 2017; 28(Suppl 5).
35 Marotta V, Nuzzo V, Ferrara T, Zuccoli A, Masone M, Nocerino L, et al. Limitations of chromogranin A in clinical practice. Biomarkers. 2012;17(2):186-91.

36 Raines D, Chester M, Diebold AE, Mamikunian P, Anthony CT, Mamikunian G, et al. A prospective evaluation of the effect of chronic proton pump inhibitor use on plasma biomarker levels in humans. Pancreas. 2012; 41(4):508-11.

37 Jensen KH, Hilsted L, Jensen C, Mynster T, Rehfeld JF, Knigge U. Chromogranin A is a sensitive marker of progression or regression in ileo-cecal neuroendocrine tumors. Scand J Gastroenterol. 2013 Jan;48(1):70-7.

38 Zatelli MC, Torta M, Leon A, Ambrosio MR Gion M, Tomassetti P, et al. Chromogranin A as a marker of neuroendocrine neoplasia: an Italian Multicenter Study. Endocr Relat Cancer. 2007;14(2):473-82.

39 Hughes MS, Azoury SC, Assadipour Y, Straughan DM, Trivedi AN, Lim RM, et al. Prospective evaluation and treatment of familial carcinoid small intestine neuroendocrine tumors (SI-NETs). Surgery. 2016; 159(1):350-6.

40 Modlin IM, Drozdov I, Kidd M. The identification of gut neuroendocrine tumor disease by multiple synchronous transcript analysis in blood. PLoS One. 2013 May;8(5):e63364.

41 Modlin IM, Drozdov I, Alaimo D, Callahan S, Teixiera N, Bodei L, et al. A multianalyte PCR blood test outperforms single analyte ELISAs (chromogranin A, pancreastatin, neurokinin A) for neuroendocrine tumor detection. Endocr Relat Cancer. 2014;21(4):615-28.

42 Pavel M, Jann H, Prasad V, Drozdov I, Modlin IM, Kidd M. NET blood transcript analysis defines the crossing of the clinical rubicon: when stable disease becomes progressive. Neuroendocrinology. 2017;104(2):170-82. 